\title{
A STRENGTHENING OF CENTERLESSNESS IN TEICHMÜLLER THEORY
}

\author{
MICHAEL ENGBER ${ }^{1}$
}

\begin{abstract}
A group-theoretic property of braid groups is used to eliminate certain hypotheses in the construction of Teichmüller space for Riemann surfaces of finite type. The result is a clarification of the geometric content of the Bers fiber space theorem.
\end{abstract}

1. Introduction. In [2], the Teichmüller space of Riemann surfaces of finite type is obtained by representing a certain functor. This generalizes Grothendieck's work [6] on the case of compact Riemann surfaces. Grothendieck's work is purely geometric and makes use of analysis only to identify his Teichmüller space with that of the analysts. This "standard of purity" was abandoned in [2] where the contractibility of Teichmüller space was used in two places in an essential way. This use compromised the claim that a purely geometric basis had been provided for the Bers fiber space theorem. The purpose of the current work is to rehabilitate that claim. All the results of [2] remain valid under the sole assumption that ordinary Teichmüller space (for compact Riemann surfaces) is simply connected. Even in the absence of this hypothesis, much can be salvaged. What makes this possible is a grouptheoretic property that is related to the property of being without a center.

I would like to thank my colleague, Peter Stebe, for his patience in answering all my questions about group theory. In addition, the proof of Theorem 1(i) for the case of a compact surface is due to him.

2. TNA groups. The following definitions suffice for our purposes although Theorem 1 is true in the more general case of braid groups with signature structure (cf. [2, Definition 1.3, p. 214]).

Definition. (i) If $f: V \rightarrow T$ is continuous, we define

$$
F_{n}(V / T)=\left\{\left(p_{1}, \ldots, p_{n}\right) \mid p_{i} \in V, f\left(p_{i}\right)=f\left(p_{j}\right), \text { all } i, j, p_{i} \neq p_{j} \text { for } i \neq j\right\}
$$

Received by the editors December 18, 1975.

AMS (MOS) subject classifications (1970). Primary 32G15, 55A05, 57A05; Secondary 20E20, 20E40, 55D10.

Key words and phrases. Teichmüller space, Riemann surface of finite type, Bers fiber space, braid group, abelian normal subgroup, contractible space.

1 This work has been partially supported by the City University Faculty Research Award Program under grant 10620. 
(ii) The symmetric group on $n$ letters, $\Sigma(n)$, acts on $F_{n}(V / T)$ by permuting entries. $B_{n}(V / T)=F_{n}(V / T) / \Sigma(n)$.

(iii) Similarly, $B_{n}^{\prime}(V / T)=F_{n}(V / T) / \Sigma(n-1)$ where the permutation group acts only on the first $n-1$ entries.

(iv) If $C$ is a topological space, let $f$ be the constant map $C \rightarrow\{p\}$. Then we write $F_{n}(C)=F_{n}(C /\{p\})$ and similarly for $B_{n}(C)$ and $B_{n}^{\prime}(C)$.

(v) A braid group of $C$ is a fundamental group of any of the spaces of (iv).

(vi) A group is said to be TNA if every abelian normal subgroup is trivial.

THEOREM 1. Let $C$ be a Riemann surface of finite type of genus at least 2. Then

(i) The fundamental group of $C$ is TNA.

(ii) The braid groups of $C$ are TNA.

Proof. (i) If $C$ has any punctures, then $\pi_{1}(C)$ is free of rank at least 2 . Let $N$ be an abelian normal subgroup. Since $N$ is abelian, $N=(1)$ or $N$ is free cyclic. If $N$ is cyclic, it must be of infinite index and being normal, it must be free on infinitely many generators [7, Theorem $2.10, \mathrm{p} .104]$.

If $C$ is compact then $\pi_{1}(C)$ is residually free [4] and centerless [7, Corollary 4.5, p. 211]. Let $N$ be an abelian normal subgroup and suppose $a \in N, a \neq 1$. Since $\pi_{1}(C)$ is centerless, there exists a $b \in \pi_{1}(C)$ such that the commutator $[a, b] \neq 1$. Choose $G$, a free quotient of $\pi_{1}(C)$ in which $\left[a^{\prime}, b^{\prime}\right] \neq 1$, where $a^{\prime}$ and $b^{\prime}$ are the images of $a$ and $b$ in $G$. Since $a^{\prime}$ and $b^{\prime}$ do not commute, rank $G \geqslant 2$. If $N^{\prime}$ denotes the image of $N$ in $G$, then $N^{\prime}$ is abelian and normal and contains $a^{\prime} \neq 1$, contradicting the first case.

To prove part (ii), we need a lemma.

LEMMA. Let $1 \rightarrow H \rightarrow G \rightarrow G^{\prime} \rightarrow 1$ be an exact sequence of groups where $H$ is TNA. Suppose either

(a) $G^{\prime}$ is TNA or

(b) $G^{\prime}$ is torsion and $G$ is torsion-free.

Then $G$ is TNA.

Proof. Let $N$ be an abelian normal subgroup of $G$. Then $H \cap N$ is normal in $H$ and abelian, whence trivial. Thus $h$ maps $N$ isomorphically onto its image $N^{\prime}$ in $G^{\prime}$. ( $h$ denotes the homomorphism $G \rightarrow G^{\prime}$.) Under hypothesis (a), $N^{\prime}$ is abelian and normal in $G^{\prime}$. Under hypothesis (b), $N^{\prime}$ is a torsion-free subgroup of the torsion group $G^{\prime}$. In either case, $N^{\prime}$ (and thus $N$ ) is trivial.

Returning to the proof of the theorem, we have exact sequences

$$
\begin{aligned}
& 1 \rightarrow \pi_{1}\left(F_{n}(C)\right) \rightarrow \pi_{1}\left(B_{n}(C)\right) \rightarrow \Sigma(n) \rightarrow 1, \\
& 1 \rightarrow \pi_{1}\left(F_{n}(C)\right) \rightarrow \pi_{1}\left(B_{n}^{\prime}(C)\right) \rightarrow \Sigma(n-1) \rightarrow 1,
\end{aligned}
$$

and $\pi_{1}\left(B_{n}(C)\right)$ and $\pi_{1}\left(B_{n}^{\prime}(C)\right)$ are torsion-free [3, pp. 117-118].

Thus it suffices to prove the result for $\pi_{1}\left(F_{n}(C)\right)$.

By [3, Theorem 3, p. 114], we have the exact sequence

$$
1 \rightarrow \pi_{1}\left(C^{\prime}\right) \rightarrow \pi_{1}\left(F_{n+1}(C)\right) \rightarrow \pi_{1}\left(F_{n}(C)\right) \rightarrow 1
$$


where $C^{\prime}$ denotes $C$ with $n$ distinct points deleted. (We shall use this notation consistently in the sequel.) Since $F_{1}(C)=C$, the result follows from part (i) by induction.

3. The Bers fiber space theorem. Let $T$ be the Teichmüller space for compact Riemann surfaces of genus $g \geqslant 2$ and let $f: V \rightarrow T$ be the universal family of Riemann surfaces over $T$ [6, exposé 7]. We will see that $T_{n}$, the Teichmüller space of Riemann surfaces of genus $g$ with $n$ punctures, is a covering space of $B_{n}(V / T)$ and that $V_{n}^{\prime}$, the family of punctured surfaces over $T_{n}$, is a covering space of $B_{n+1}^{\prime}(V / T)$. This leads to a weak form of the Bers fiber space theorem. If we adjoin the hypothesis that $T$ is simply connected, then the theorem is true in its strong form [2, Corollary 4.3, p. 225].

We know from [6, expose $17, \S 5]$ that the mapping $f: V \rightarrow T$ is smooth and proper and that $T$ is a manifold so that $f$ is topologically (even $C^{\infty}$ ) a locally trivial fibration.

Proposition. Let $C$ be a compact surface of genus $g$. Then

$$
1 \rightarrow \pi_{1}\left(B_{n}(C)\right) \rightarrow \pi_{1}\left(B_{n}(V / T)\right) \rightarrow \pi_{1}(T) \rightarrow 1
$$

is exact and similarly for $B_{n}^{\prime}$.

Proof. Since $V \rightarrow T$ is locally trivial with fiber $C$, it follows that $B_{n}(V / T)$ $\rightarrow T$ is locally trivial with fiber $B_{n}(C)$. Applying the exact homotopy sequence of a fibration, we get

$$
\pi_{2}\left(B_{n}(V / T)\right) \stackrel{g}{\rightarrow} \pi_{2}(T) \rightarrow \pi_{1}\left(B_{n}(C)\right) \stackrel{h}{\rightarrow} \pi_{1}\left(B_{n}(V / T)\right) \rightarrow \pi_{1}(T) \rightarrow 1 .
$$

By exactness at $\pi_{1}\left(B_{n}(C)\right)$, we get ker $h \cong \operatorname{coker} g$. But as a quotient of an abelian group, coker $g$ is abelian and, since it is isomorphic to ker $h$, we have an abelian normal subgroup of $\pi_{1}\left(B_{n}(C)\right)$. Since this last is TNA, $\operatorname{ker} h$ is trivial.

In the absence of knowledge that $T$ is simply connected, we cannot identify $\pi_{1}\left(B_{n}(C)\right)$ with $\pi_{1}\left(B_{n}(V / T)\right)$. It is the identification of these two groups [2, Proposition 3.5, p. 222] which permits the conclusion that $T_{n}$ is the universal covering space of $B_{n}(V / T)$ [2, Theorem 3.6, p. 222]. Nonetheless, [6, exposé 7, Proposition 8.3] or [2, Proposition 3.3, p. 221] implies that $T_{n}$ is a covering space of $B_{n}(V / T)$ and the arguments of $[2, \S 4]$ give analogous results for $V_{n}^{\prime}$.

We summarize in the following theorem.

THEOREM 2. $T_{n}$ is a covering space of $B_{n}(V / T)$ and $V_{n}^{\prime}$ is a covering space of $B_{n+1}^{\prime}(V / T)$. If $T$ is simply connected, then $T_{n}$ is the universal cover of $B_{n}(V / T)$ and $V_{n}^{\prime}$ is the covering space of $B_{n+1}^{\prime}(V / T)$ associated to the subgroup $\pi_{1}\left(C^{\prime}\right)$ of $\pi_{1}\left(B_{n+1}^{\prime}(V / T)\right) \cong \pi_{1}\left(B_{n+1}^{\prime}(C)\right)$.

REMARK. The identification of $\pi_{1}\left(C^{\prime}\right)$ as a subgroup of $\pi_{1}\left(B_{n+1}^{\prime}(C)\right)$ is a result of the exact sequence [3, Theorem 3, p. 114] 


$$
1 \rightarrow \pi_{1}\left(C^{\prime}\right) \rightarrow \pi_{1}\left(B_{n+1}^{\prime}(C)\right) \rightarrow \pi_{1}\left(B_{n}(C)\right) \rightarrow 1 .
$$

COROLlary (BERs). The universal covering space of $T_{n+1}$ is isomorphic to the universal covering space of $V_{n}^{\prime}$.

4. Constancy of homotopy type. In what follows, $T_{0}=T$.

THEOREM 3. $\pi_{i}\left(T_{n+1}\right) \cong \pi_{i}\left(T_{n}\right)$ for all $i \geqslant 2$ and all $n \geqslant 0$. If $T$ is simply connected then the morphism $F: T_{n+1} \rightarrow T_{n}$ defined by forgetting one of the punctures is a homotopy equivalence for all $n$.

Proof. Since $T_{n+1}$ and $V_{n}^{\prime}$ have the same universal cover, $\pi_{i}\left(T_{n+1}\right) \cong \pi_{i}\left(V_{n}^{\prime}\right)$ for $i \geqslant 2$. On the other hand, $f_{n}: V_{n}^{\prime} \rightarrow T_{n}$ is a locally trivial fibration with fiber $C^{\prime}$ for all $n$. From the homotopy sequence of this fibration,

$$
\pi_{i}\left(C^{\prime}\right) \rightarrow \pi_{i}\left(V_{n}^{\prime}\right) \rightarrow \pi_{i}\left(T_{n}\right) \rightarrow \pi_{i-1}\left(C^{\prime}\right)
$$

is exact. Since $\pi_{i}\left(C^{\prime}\right)=0$ for $i \geqslant 2, f_{n}$ induces an isomorphism for $i \geqslant 3$. For $i=2$, we have

$$
0 \rightarrow \pi_{2}\left(V_{n}^{\prime}\right) \stackrel{g}{\rightarrow} \pi_{2}\left(T_{n}\right) \rightarrow \pi_{1}\left(C^{\prime}\right) \stackrel{h}{\rightarrow} \pi_{1}\left(V_{n}^{\prime}\right),
$$

where $g$ is induced by $f_{n}$. An argument exactly like that of the Proposition shows that coker $g=0$. Thus $\pi_{i}\left(V_{n}^{\prime}\right) \cong \pi_{i}\left(T_{n}\right), i \geqslant 2$. If $T$ is simply connected, then $T_{n+1}$ is the universal cover of $V_{n}^{\prime}$ so the isomorphisms $\pi_{i}\left(T_{n+1}\right)$ $\cong \pi_{i}\left(V_{n}^{\prime}\right), i \geqslant 2$, are induced by the covering map $T_{n+1} \rightarrow V_{n}^{\prime}$. Furthermore, $\pi_{1}\left(T_{n+1}\right)=\pi_{1}\left(T_{n}\right)=1$. Thus the composite morphism $T_{n+1} \rightarrow V_{n}^{\prime} \rightarrow T_{n}$ induces isomorphisms on all homotopy groups. Since the $T_{n}$ are manifolds, this map is a homotopy equivalence. That the composite morphism is forgetting one puncture can be seen by [2, Proposition 4.1, p. 224].

In the geometric framework, this result corresponds to the well-known contractibility of Teichmüller spaces. The weaker statement that $T$ is simply connected is of a different character. Grothendieck has shown [6, exposé 7, Proposition 8.5] that there exists a unique simply connected moduli space for rigidified (i.e., "marked") Riemann surfaces. Furthermore, the corresponding rigidification is uniquely determined. The contractibility of the Teichmüller space is then used only to show that this simply connected space is the Teichmüller space. The remainder of Grothendieck's program has also been achieved [6, exposé 7, Remark 3.2, $3^{\circ}$, p. 7-09]. Earle and Eells have shown [1, $8 \mathrm{D}$, Remark 2, p. 34] that the contractibility of Teichmüller space is equivalent to that of the group of diffeomorphisms of $C$ homotopic to the identity. A topological proof of the contractibility of this diffeomorphism group has been found by A. Gramain [5].

\section{BIBLIOGRAPHY}

1. C. J. Earle and J. Eells, A fibre bundle description of Teichmüller theory, J. Differential Geometry 3 (1969), 19-43. MR 43 \#2737a. 
2. M. Engber, Teichmüller spaces and representability of functors, Trans. Amer. Math. Soc. 201 (1975), 213-226.

3. E. Fadell and L. Neuwirth, Configuration spaces, Math. Scand. 10 (1962), 111-118. MR 25 \#4537.

4. K. N. Frederick, The Hopfian property for a class of fundamental groups, Comm. Pure Appl. Math. 16 (1963), 1-8. MR 26 \#6948.

5. A. Gramain, Le type d'homotopie du groupe des difféomorphismes d'une surface compacte, Ann. Sci. École Norm. Sup. (4) 6 (1973), 53-66. MR 48 \#5116.

6. A. Grothendieck, Techniques de construction en géométrie analytique, Séminaire H. Cartan, 1960/61, exposés 7, 9-17, Secrétariat mathématique, Paris, 1962.

7. W. Magnus, A. Karrass and D. Solitar, Combinatorial group theory. Presentations of groups in terms of generators and relations, Pure and Appl. Math., vol. 13, Interscience, New York, 1966. MR 34 \# 7617.

Department of Mathematics, City College, CUNY, New York, New York 10031 